\title{
Expression of Matrix Metalloproteinase-1 in Alveolar Macrophages, Type II Pneumocytes, and Airways in Smokers: Relationship to Lung Function and Emphysema
}

\author{
Alison M. Wallace • Leanna B. Loy • Raja T. Abboud • Jeanine M. D'Armiento • \\ Harvey O. Coxson • Nestor L. Muller $\cdot$ Steve Kalloger $\cdot$ Xing Li • \\ W. Mark Elliott · John C. English $\cdot$ Richard J. Finley $\cdot$ Peter D. Paré
}

Received: 6 December 2013/Accepted: 7 April 2014/Published online: 3 May 2014

(c) The Author(s) 2014. This article is published with open access at Springerlink.com

\begin{abstract}
Background An imbalance between proteolytic enzymes and their inhibitors is thought to be involved in the pathogenesis of chronic obstructive pulmonary disease. Matrix metalloproteinase-1, also known as interstitial collagenase, has been implicated as a potentially important proteinase in the genesis of chronic obstructive pulmonary disease and, more specifically, emphysema.

Methods We performed quantitative immunohistochemical assessment of matrix metalloproteinase- 1 expression in the resected lung of 20 smokers/ex-smokers who had varying severity of airflow obstruction and emphysema and compared this with the lungs of 5 nonsmokers. Emphysema was measured using a morphometric measure of the lungs' surface area/volume ratio and with qualitative and quantitative computed tomography (CT) measures of emphysema. Results There were significantly more matrix metalloproteinase-1-expressing alveolar macrophages and type II
\end{abstract}

Electronic supplementary material The online version of this article (doi:10.1007/s00408-014-9585-6) contains supplementary material, which is available to authorized users.

A. M. Wallace · L. B. Loy · H. O. Coxson · X. Li ·

W. Mark Elliott · P. D. Paré $(\square)$

St. Paul's Hospital, University of British Columbia Center for

Heart Lung Innovation, 166 - 1081 Burrard Street, Vancouver,

BC V6Z 1Y6, Canada

e-mail: Peter.Pare@hli.ubc.ca

A. M. Wallace · J. M. D'Armiento

Division of Molecular and Pulmonary Medicine, Department of

Medicine, College of Physicians and Surgeons, Columbia

University, New York, NY, USA

R. T. Abboud · P. D. Paré

Division of Respiratory Medicine, Department of Medicine,

University of British Columbia, Vancouver, BC, Canada pneumocytes as well as a greater percentage of small airways that stained positively for matrix metalloproteinase- 1 in the lungs of smokers than in those of nonsmokers $(p<0.0001, p<0.0001$, and $p=0.0003$, respectively). The extent of staining of type II pneumocytes and airways for matrix metalloproteinase-1 was significantly related to the extent of smoking ( $p=0.012$ and $p=0.013$, respectively). In addition, the extent of matrix metalloproteinase1 staining of alveolar macrophages was related to the lung surface area/volume ratio and to qualitative estimates of emphysema on CT.

Conclusion These findings suggest that cigarette smoking increases expression of matrix metalloproteinase- 1 in alveolar macrophages as well as in alveolar and small airway epithelial cells. Smokers who develop emphysema have increased alveolar macrophage expression of matrix metalloproteinase-1.

Keywords Computed tomography · Emphysema · Expression - Immunohistochemistry - Metalloproteinase · Lung

H. O. Coxson · N. L. Muller · S. Kalloger

Department of Radiology, Vancouver General Hospital,

University of British Columbia, Vancouver, BC, Canada

J. C. English

Department of Pathology, Vancouver General Hospital,

University of British Columbia, Vancouver, BC, Canada

R. J. Finley

Department of Surgery, Vancouver General Hospital, University

of British Columbia, Vancouver, BC, Canada 


\section{Introduction}

Cigarette smoke is the primary risk factor for chronic obstructive pulmonary disease (COPD), a disease state characterized by airflow limitation that is not fully reversible [1]. Not everyone who smokes develops COPD, and when it does occur, the pathological changes vary between patients and at different stages of the disease. The variable susceptibility and phenotypes suggest that there is a genetic component to the disease. Pathologic changes characteristic of COPD are found in the large airways, small airways $(<2 \mathrm{~mm})$, and alveoli. Chronic inflammation leads to fixed narrowing and obliteration of small airways and alveolar wall destruction; both changes result in airflow limitation.

More than 30 years ago, Hogg et al. [2] proposed that COPD is primarily a disease of the small airways based on the observation that the peripheral airways are the major site of increased resistance to airflow. Changes that contribute to the narrowing of the small airways include loss of alveolar and bronchiolar attachments, goblet-cell metaplasia, edema, inflammatory cellular infiltration, reduced surfactant, smooth muscle hyperplasia, and fibrosis [3]. The narrowing and obliteration of the small airways coupled with the loss of lung elastic recoil caused by parenchymal destruction leads to expiratory airflow limitation. The destruction of the lung parenchyma, which causes the lesions of emphysema, is believed to be due to an imbalance between the proteolytic and antiproteolytic processes in the lung. A number of proteolytic enzymes have been implicated in the disease process, including matrix metalloproteinases (MMPs), a family of zinc-dependent proteinases with the capacity to degrade both elastin and collagen.

MMP-1 is one of the most abundant proteases in the MMP family and is capable of degrading type I, II, and III collagens. Type I and III collagens are the most abundant proteins within the lung. The finding that MMP-1 transgenic mice overexpressing MMP-1 develop emphysematous changes in their lungs showed a direct contribution of MMPs to this proteolytic destruction [4, 5]. Further evidence from animal and human studies supports a role for collagenolytic enzymes and, in particular, MMP-1 in the pathogenesis of emphysema [6-9]. Genetic studies have also shown that MMP-1 polymorphisms are associated with COPD phenotypes [10, 11]. Alveolar macrophages, type II pneumocytes, and airway epithelial cells are known to express MMP-1 [7, 9]. In vitro studies have shown that cigarette smoke directly targets the MMP-1 promoter in human small-airway epithelial cells [12] and that this increase in expression is regulated via the extracellular regulated kinase/mitogen-activated protein kinase pathway [13]. Individual sensitivity to upregulation of expression of
MMP-1 in response to cigarette smoke could contribute to individual susceptibility for COPD among smokers. Imai et al. [7] have previously identified alveolar macrophages and type II pneumocytes as two important cell types in the lung responsible for MMP-1 expression. Unfortunately, the study was limited by the small number of subjects (only two) who were long-term smokers but had normal lung tissue and therefore they were not able to compare the expression of MMP-1 in smokers with and without emphysema.

In the present study we quantified MMP-1 expression within the lung by immunohistochemistry and related MMP-1 expression to histological and computed tomography (CT) measures of emphysema in order to better evaluate the pathogenic role of this enzyme.

\section{Methods}

Human lung specimens were obtained from 25 patients. Twenty lobes or lungs were obtained from smokers at the time of surgical resection for small $(<3 \mathrm{~cm}$ diameter), stage I or II, peripheral tumors at the Vancouver General Hospital in Vancouver. Study participants who quit smoking 6 months or more prior to surgery were considered former smokers $(n=12)$; the remaining subjects in the smoking group were classified as current smokers $(n=8)$. The lungs or lobes of five never-smokers were obtained as controls from individuals who had surgical resection for various indications at St. Paul's Hospital in Vancouver. Within the week prior to surgery the patients completed a questionnaire regarding smoking and respiratory symptoms and had measurements of subdivisions of lung volume, spirometry, and single-breath diffusing capacity as previously described and conforming to ATS standards [14]. Subjects provided informed consent. The study was approved by the University of British Columbia/ Providence Health Care and Vancouver General Hospital Research Ethics Boards. Statistical analysis was performed using R 2.4.0 (Auckland, NZ). As a quantitative estimate of lifetime exposure to cigarette smoke, we used pack-years as ascertained in the preoperative interview. Unpaired $t$ tests were used to compare anthropometric, physiological, and morphological data of never-smokers with those of smokers and to make comparisons between former smokers and current smokers. Simple linear regression was used to estimate the associations between MMP-1 staining (alveolar macrophages, type II pneumocytes, and airways) and morphometric estimates of emphysema $(\mathrm{Sa} / \mathrm{V})$, as well as the qualitative and quantitative $\mathrm{CT}$ values. $P$ values less than 0.05 were considered statistically significant. Details of the immunohistochemistry, image analysis, and CT analysis can be found in the Online Resource. 
Table 1 Characteristics of study subjects

\begin{tabular}{lll}
\hline & $\begin{array}{l}\text { Never-smokers } \\
(n=5)\end{array}$ & $\begin{array}{l}\text { Smokers } \\
(n=20)\end{array}$ \\
\hline Sex (male/female) & $2 / 3$ & $13 / 7$ \\
Age (years) & $55.2 \pm 12.4$ & $68.7 \pm 11.0$ \\
Smoking history (pack years) & $0 \pm 0.0$ & $52.6 \pm 33.3$ \\
FEV $_{1} \%$ predicted & $109.0 \pm 16.0$ & $79.0 \pm 16.5$ \\
DLCO \% predicted & $89.0 \pm 4.5$ & $75.0 \pm 11.3$ \\
\hline
\end{tabular}

Data are mean \pm SEM

\section{Results}

\section{Patient Characteristics}

The demographics, smoking history, and pulmonary function of the subjects are presented in Table 1. In our sample, the never-smokers were significantly younger than the smokers $(p=0.027)$. Pulmonary function tests showed that the smokers had a reduced forced expiratory volume in 1 second $\left(\mathrm{FEV}_{1}\right)$ compared with the never-smokers $(p=0.002)$ and a lower diffusing capacity for carbon monoxide $(p=0.033)$.

\section{Cellular Localization of MMP-1}

Immunohistochemistry was undertaken to identify the cell type(s) responsible for MMP-1 expression within the lung. MMP-1 was immunolocalized to alveolar macrophages (Fig. 1a), type II pneumocytes (Fig. 1a, b), and airways (Fig. 1d). Negative controls are shown in Fig. 1c, e.

Comparison of MMP-1-Positive Cells in the Never-Smoker and Smoker Groups

Table 2 shows the amount of MMP-1-positive cells in the never-smoker and smoker groups. Smokers had significantly more MMP-1-positive stained alveolar macrophages $(p<0.0001)$, type II pneumocytes $(p<0.0001)$, and airways $(p<0.0003)$. Type II pneumocytes did not stain positively for MMP-1 in the lungs of never-smokers, staining was weakly positive in alveolar macrophages, and only one nonsmoker had any positive airway staining.

To determine if the amount of MMP-1 staining and qualitative CT score were affected by current smoking status, we divided the smoking group into former smokers and current smokers. Table 3 shows that there were no significant differences between the two subgroups in terms of the amount of MMP-1 staining in any of the cell types,

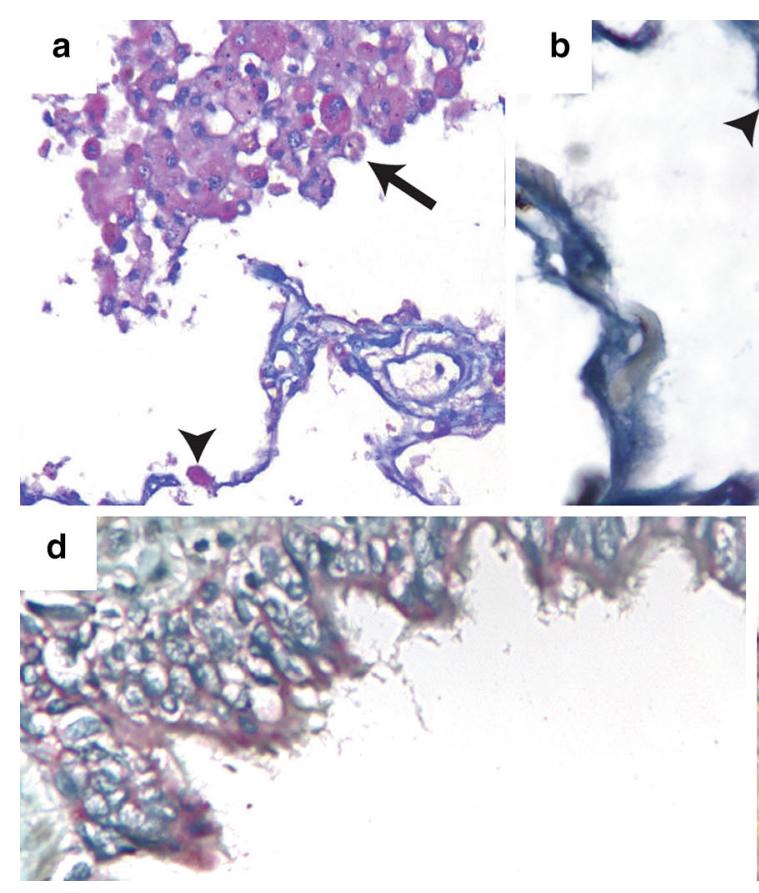

Fig. 1 Immunostaining of the lungs of smokers with emphysema and never-smoker control subjects was performed with the use of a mouse monoclonal antibody specific to human MMP-1. Lung tissue from a smoker with emphysema showing positive MMP-1 staining in alveolar macrophages (a) and type II pneumocytes (b). Panel

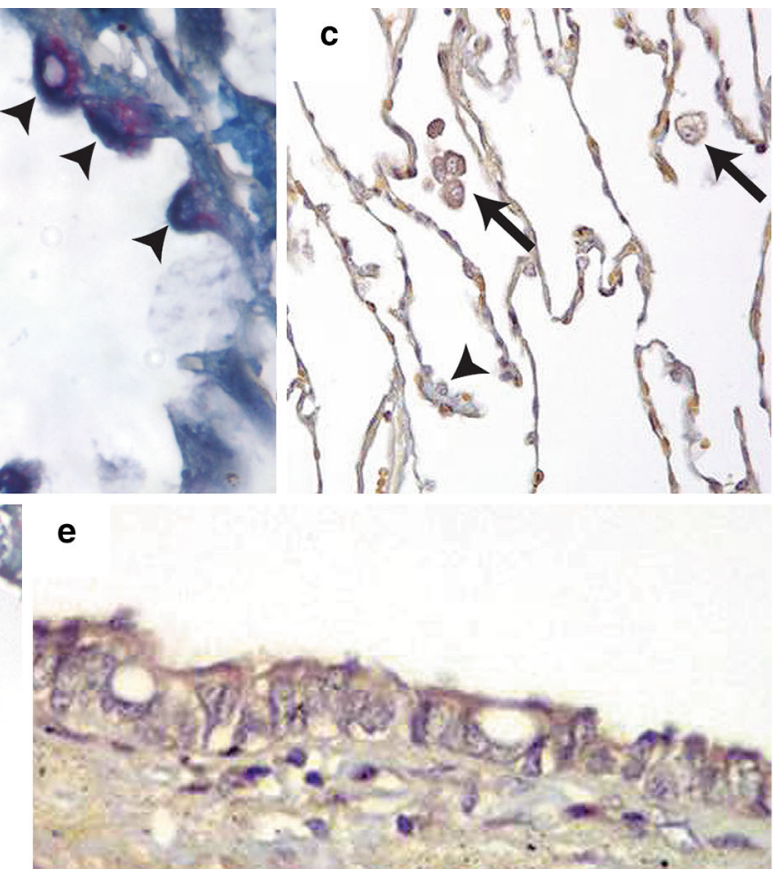

c shows a macrophage and a type II pneumocyte negative control. Panel d shows MMP-1-positive airway epithelial cells and panel e shows negative airway epithelium. Arrows indicate alveolar macrophage staining for MMP-1. Arrowheads indicate type II pneumocyte staining for MMP-1 $(20 \times$ for all pictures $)$ 
Table 2 MMP-1 positivity in the never-smoker and smoker groups

\begin{tabular}{lccr}
\hline & $\begin{array}{l}\text { Never-smokers } \\
(n=5)\end{array}$ & $\begin{array}{l}\text { Smokers } \\
(n=20)\end{array}$ & $p$ value \\
\hline Alveolar macrophages & $1.38^{\mathrm{a}} \pm 0.60$ & $9.68^{\mathrm{a}} \pm 1.45$ & $<0.0001$ \\
Type II pneumocytes & $0.0 \pm 0.0$ & $0.78^{\mathrm{a}} \pm 0.17$ & $<0.0001$ \\
Airways & $10 \pm 10$ & $77 \pm 6$ & 0.0003 \\
\hline
\end{tabular}

Data are mean \pm SEM. The values for alveolar macrophages and type II pneumocytes represent the volume fractions of positively staining cells over total lung volume

a The volume fractions have been multiplied by $10^{6}$ for convenience

b MMP-1 positivity of airways is expressed as the percentage of the airways positive for MMP-1

suggesting that smoking cessation does not result in a decrease in MMP-1 expression. In addition, the quantitative CT score did not differ significantly between the former smokers and current smokers (Table 3).

\section{Amount of Staining and Emphysema}

We analyzed whether staining in each of the cell types was related to the surface area/volume ratio (a low ratio indicates emphysema), as well as to the qualitative and quantitative CT grades of emphysema. Since smoking had such a profound effect on MMP-1 expression, we limited the analysis to the 20 subjects with a smoking history. This addresses the question of whether smokers who develop emphysema have increased expression of MMP-1 relative to smokers who do not. The surface area/volume ratio was significantly negatively related to alveolar macrophage staining ( $r=-0.43, p=0.029)$ but not to type II pneumocyte staining $(r=-0.29, p=0.11)$ or airway staining $(r=-0.25, p=0.14)$.

The distribution of qualitative CT emphysema scores was grade $0(n=1)$, grade $1(n=9)$, grade $2(n=6)$, and grade $4(n=1)$. For ease of comparison, the one subject with grade 0 and the one subject with grade 4 were excluded from the analysis; there were no subjects in the grade 3 group. The amount of MMP-1 staining in alveolar macrophages in subjects with grade 2 emphysema was significantly greater than that of those with grade 1 emphysema $(p=0.03)$. There was no significant difference between the amounts of staining in type II pneumocytes and airways as a function of the qualitative grade of emphysema. There were also no significant associations between quantitative CT values and the amount of positively stained cells. When adjusted for smoking status, the overall emphysema score (defined using the density mask cutoff of -855 Hounsfield units) showed a borderline association with staining of type II pneumocytes $(p=0.073)$, with higher emphysema being positively related to staining for MMP-1.

\section{Discussion}

The results of this study provide some support for the claim by D'Armiento et al. [7] that MMP-1, a nonelastolytic collagenase, may play a pathogenic role in emphysema. In addition, the results are concordant with our previous finding of a significant relationship between MMP-1 mRNA expression by alveolar macrophages and the extent of emphysema measured by CT scan [9]. In the present study we demonstrated MMP-1 immunolocalization to alveolar macrophages, type II pneumocytes, and airways. Furthermore, MMP-1 staining was significantly increased in smokers; in the control group there was no staining of type II pneumocytes and only one of five individuals showed any staining of airway epithelium. Interestingly, the amount of MMP-1 staining did not differ between the current and former smokers, suggesting that cigarette smoke induces long-term changes in the expression of this proteinase that persist after smoking cessation. Although

Table 3 Staining, expressed as volume fraction and percent positive airways, and quantitative CT scores in the smoking group according to smoking status

\begin{tabular}{llll}
\hline & Former smokers $^{\mathrm{a}}(n=12)$ & Current smokers $(n=8)$ & $p$ value \\
\hline Alveolar macrophages & $10.68^{\mathrm{b}} \pm 0.195$ & $8.19^{\mathrm{b}} \pm 0.218$ & 0.203 \\
Type II pneumocytes & $0.86^{\mathrm{b}} \pm 0.25$ & $6.62^{\mathrm{b}} \pm 0.23$ & 0.293 \\
Airways & $84 \pm 4$ & $67 \pm 12$ & 0.073 \\
Overall emphysema \% $^{\mathrm{c}}$ & $25.1(14.3-31.7)$ & $45.4(32.8-49.7)$ & 0.234 \\
Severe emphysema ${ }^{\mathrm{d}}$ & $2.3(0.4-13.4)$ & $8.1(4.9-21.3)$ & 0.181 \\
\hline
\end{tabular}

Data are mean \pm SEM or (interquartile range)

a Study participants who quit smoking 6 months or more prior to surgery

b The volume fractions have been multiplied by $10^{6}$ for convenience

c Defined using a density mask cutoff of -855 Hounsfield units

${ }^{\mathrm{d}}$ Defined using a density mask cutoff of -910 Hounsfield units 
MMP-1 staining was increased in all smokers, there was significantly more staining of alveolar macrophages in individuals with a higher emphysema score. This is a novel observation. Previously, when MMP-1 expression in the lungs of smokers with emphysema was compared to that of nonsmokers, substantial differences were found in MMP-1 expression by alveolar macrophages and type II pneumocytes [7]. However, only two smokers without emphysema were included in the study and thus the contribution of smoking versus emphysema could not be distinguished.

The inflammatory mediators and proteases that initiate and sustain proteolytic injury to the lung remain unclear. Multiple studies have shown that the numbers of inflammatory cells in the lung are markedly increased in response to cigarette smoke exposure [15]. Our findings show that the macrophages in smokers' lungs express increased MMP-1 and, furthermore, that the number of positive macrophages increases with the severity of emphysema. However, it is not known whether this initial inflammatory reaction is harmful or to what extent resultant upregulation of other proteolytic enzymes also causes lung destruction. For example, the expression of MMP-12, an elastolytic enzyme, has also been shown to be increased in individuals with emphysema [9]. Although transgenic upregulation of MMP-1 in mice leads to emphysema [4], knockout of the MMP-12 gene prevents the development of emphysema in mice exposed to cigarette smoke [16], implicating both of these proteases in the process. In this study we focused on MMP-1 because of its ability to degrade type III collagen, the major structural component of the alveolar ducts and septa, which are key regions subjected to proteolytic destruction in emphysema [8].

Our results are also supported by the study of Gosselink et al. [17]. Those investigators used laser capture microdissection to obtain mRNA from the alveolar region of the peripheral lung as well as from the small airways. They showed that MMP-1 expression was increased in the lung parenchymal tissue as a function of increasing GOLD COPD severity. There was no differential expression of MMP-1 in the airways as a function of airflow obstruction.

This study has limitations. It is an observational study examining the expression of MMP-1 within various cell types within the lung in a small sample of subjects. We measured the volume fraction of the lung parenchyma that contained positive staining alveolar macrophages and type II pneumocytes rather than the fraction of these cell types that were positive. Therefore, it is possible that an increase in the total number of alveolar macrophages and type II pneumocytes in the lungs of the smokers and in the more emphysematous subjects could contribute to the differences. Although it is true that there are more alveolar macrophages and type II pneumocytes in the lungs of smokers, we do not believe that this is the main source of the difference. The alveolar macrophages and type II pneumocytes of nonsmokers displayed little or no staining; the signal was not due simply to increased cell number. Similarly, there were more MMP-1-positive alveolar macrophages and type II pneumocytes in subjects with more severe emphysema, not simply more cells.

As discussed above, a potential weakness of this study is that we examined the expression of only one enzyme thought to play a destructive role in the disease process. Furthermore, due to our limited sample size, we were not able to fully examine the relationship between MMP-1 expression and all of the GOLD stages of disease or grades of emphysema. Our never-smoker controls were younger than our smokers, with and without emphysema, primarily due to the difficulty of obtaining normal samples from patients in their fifth and sixth decade of life, when emphysema normally affects patients.

In summary, our findings further support the proposal that inflammatory cells (alveolar macrophages), as well as type II pneumocytes and airway epithelium, are a significant source of MMP-1 and that this enzyme plays a role in the pathogenesis of emphysema, even after smoking cessation. In addition, increased MMP-1 expression correlates with more severe disease, suggesting that MMP-1 plays a role in the disease process and that regulation of this enzyme may prove to be a valuable therapeutic intervention.

Acknowledgments The authors thank all participants for their contribution to the study. The study was supported in part by NIH Grant R01:HL086936 (JD). AW received funding from the British Columbia Clinical Genomics Network.

Conflict of interest The authors have no conflicts of interest to disclose.

Open Access This article is distributed under the terms of the Creative Commons Attribution License which permits any use, distribution, and reproduction in any medium, provided the original author(s) and the source are credited.

\section{References}

1. Pauwels RA, Buist AS, Calverley PM, Jenkins CR, Hurd SS (2001) Global strategy for the diagnosis, management, and prevention of chronic obstructive pulmonary disease. NHLBI/WHO global initiative for chronic obstructive lung disease (GOLD) workshop summary. Am J Respir Crit Care Med 163:1256-1276

2. Hogg JC, Macklem PT, Thurlbeck WM (1968) Site and nature of airway obstruction in chronic obstructive lung disease. N Engl J Med 278:1355-1360

3. Cherniack NS (1991) Chronic obstructive pulmonary disease. W.B. Saunders, Toronto

4. D'Armiento J, Dalal SS, Okada Y, Berg RA, Chada K (1992) Collagenase expression in the lungs of transgenic mice causes pulmonary emphysema. Cell 71:955-961

5. Foronjy RF, Okada Y, Cole R, D'Armiento J (2003) Progressive adult-onset emphysema in transgenic mice expressing human 
MMP-1 in the lung. Am J Physiol Lung Cell Mol Physiol 284:L727-L737

6. Segura-Valdez L, Pardo A, Gaxiola M, Uhal BD, Becerril C, Selman M (2000) Upregulation of gelatinases A and B, collagenases 1 and 2, and increased parenchymal cell death in COPD. Chest 117:684-694

7. Imai K, Dalal S, Chen E, Downey R, Schulman L, Ginsburg M, D'Armiento J (2001) Human collagenase (matrix metalloproteinase-1) expression in the lungs of patients with emphysema. Am J Respir Crit Care Med 163:786-791

8. Shiomi T, Okada Y, Foronjy R, Schiltz J, Jaenish R, Krane S, D'Armiento J (2003) Emphysematous changes are caused by degradation of type III collagen in transgenic mice expressing MMP-1. Exp Lung Res 29:1-15

9. Wallace AM, Sandford AJ, English JC, Burkett KM, Li H, Finley RJ, Müller NL, Coxson HO, Paré PD, Abboud RT (2008) Matrix metalloproteinase expression by human alveolar macrophages in relation to emphysema. COPD 5:13-23

10. Joos L, He JQ, Shepherdson MB, Connett JE, Anthonisen NR, Paré PD, Sandford AJ (2002) The role of matrix metalloproteinase polymorphisms in the rate of decline in lung function. Hum Mol Genet 11:569-576

11. DeMeo DL, Hersh CP, Hoffman EA, Litonjua AA, Lazarus R, Sparrow D, Benditt JO, Criner G, Make B, Martinez FJ, Scanlon
PD, Sciurba FC, Utz JP, Reilly JJ, Silverman EK (2007) Genetic determinants of emphysema distribution in the national emphysema treatment trial. Am J Respir Crit Care Med 176:42-48

12. Mercer BA, Wallace AM, Brinckerhoff CE, D'Armiento JM (2009) Identification of a cigarette smoke-responsive region in the distal MMP-1 promoter. Am J Respir Cell Mol Biol 40:4-12

13. Mercer BA, Kolesnikova N, Sonett J, D’Armiento J (2004) Extracellular regulated kinase/mitogen activated protein kinase is up-regulated in pulmonary emphysema and mediates matrix metalloproteinase-1 induction by cigarette smoke. J Biol Chem 279:17690-17696

14. Hogg JC, Wright JL, Wiggs BR, Coxson HO, Opazo Saez A, Paré PD (1994) Lung structure and function in cigarette smokers. Thorax 49:473-478

15. Hogg JC (1980) Structure and function of small airways. Chest $77(2$ Suppl):279-282

16. Hautamaki RD, Kobayashi DK, Senior RM, Shapiro SD (1997) Requirement for macrophage elastase for cigarette smokeinduced emphysema in mice. Science 277:2002-2004

17. Gosselink JV, Hayashi S, Elliott WM, Xing L, Chan B, Yang L, Wright C, Sin D, Paré PD, Pierce JA, Pierce RA, Patterson A, Cooper J, Hogg JC (2010) Differential expression of tissue repair genes in the pathogenesis of chronic obstructive pulmonary disease. Am J Respir Crit Care Med 181:1329-1335 